\title{
AXIALLY ASYMMETRIC STRESSES IN A TRANSVERSELY ISOTROPIC, SHORT CYLINDER SUBJECTED TO SECTORIAL PRESSURES ON THE END FACES
}

Isamu A. OKUMURA*

\begin{abstract}
An analysis of axially asymmetric stresses in a transversely isotropic, short cylinder subjected to sectorial pressures on the end faces is presented. The generalized Elliott solution is used for the analysis. The solution yields two different elasticity solutions to be necessary for satisfying boundary conditions at the end faces and the side surface. Magnesium and cadmium crystals and an isotropic material are treated. Numerical results for displacements and stresses in these materials are illustrated. The effect of anisotropy on the displacements and stresses is examined through a comparison with the isotropic material.

Key Words : elasticity, transverse isotropy, stress analysis, asymmetric problem, short cylinder
\end{abstract}

\section{INTRODUCTION}

Recent studies on three-dimensional elasticity problems have turned to those of anisotropic solids. Although there are various classes of anisotropy, practical necessity is concerned with orthotropy, cylindrical anisotropy or transverse isotropy. Studies on orthotropic and cylindrically anisotropic solids are few in number at the present time, because it is hard to find the three-dimensional elasticity solutions to these anisotropic solids. However, there are a lot of studies on transversely isotropic solids, because the three-dimensional elasticity solutions to this anisotropy have been found. Levine and Klosner ${ }^{1)}$ analyzed axially symmetric stresses in a long cylindrical shell subjected to radial band loads. Atsumi and Itou ${ }^{2)}$ analyzed axially symmetric stresses in an infinite cylinder with a spherical cavity. Mirsky ${ }^{3)}$ and $\mathrm{Chen}^{4)}$ analyzed the wave propagation in an infinite cylinder and the concentrated force moving with uniform velocity in an infinite solid, respectively. Zureick $^{5}$ analyzed axially asymmetric stresses in an infinite solid containing a spheroidal cavity.

These studies as stated above are concerned with infinite solids and use Elliott's and Lodge's solutions. Although Elliott's and Lodge's solutions are simple in applications, they seem to have been hardly applied to three-dimensional problems of finite solids, for instance, short rectangular prisms, short cylinders or short hollow cylinders. The writer proposed the generalized Elliott solution and analyzed axially symmetric stresses in a short

\footnotetext{
* Member of JSCE, Dr. Eng., Professor, Dept. of Civ. Eng., Kitami Institute of Technology (165 Koen-cho, Kitami 090, JAPAN)
}

cylinder subjected to a radial band load in a previous paper ${ }^{6}$. The solution was also applied to an axially symmetric stress analysis of a short hollow cylinder subjected to an outer band load ${ }^{7}$. However, studies on axially asymmetric stresses in a short cylinder or in a short hollow cylinder have not, to the writer's knowledge, been carried out.

This paper is concerned with an analysis of axially asymmetric stresses in a transversely isotropic, short cylinder subjected to sectorial pressures on the end faces. The generalized Elliott solution is used for the analysis. The threedimensional problem of a short cylinder is much more complicated than that of an infinite or a long cylinder, because the method of solution requires two different elasticity solutions to satisfy boundary conditions at the end faces and the side surface. The generalized Elliott solution yields the two elasticity solutions. Additional solutions are also used to treat initial terms in Fourier and Bessel expansions. Magnesium and cadmium crystals, as examples of transversely isotropic materials, as well as an isotropic material, are treated in numerical calculations.

\section{THE GENERALIZED ELLIOTT SOLUTION}

Using cylindrical coordinates $(r, \theta, z)$ such that the $z$-axis is taken parallel to the axis of elastic symmetry, the generalized Elliott solution ${ }^{6}$ is expressed in terms of displacement components, i.e., $u_{r}, u_{\theta}$ and $u_{z}$ as

$$
\begin{aligned}
u_{r}= & \frac{\partial}{\partial r}\left[\phi_{01}+\phi_{03}+\gamma_{1}\left(r \frac{\partial \phi_{1}}{\partial r}+z \frac{\partial \phi_{3}}{\partial z}\right)\right. \\
& \left.-\gamma_{2} \phi_{1}-\gamma_{3} \phi_{3}\right]+\frac{1}{r} \frac{\partial \phi}{\partial \theta} \ldots \ldots \ldots \ldots
\end{aligned}
$$




$$
\begin{aligned}
& u_{\theta}=\frac{1}{r} \frac{\partial}{\partial \theta}\left[\phi_{01}+\phi_{03}+\gamma_{1}\left(r \frac{\partial \phi_{1}}{\partial r}+z \frac{\partial \phi_{3}}{\partial z}\right)\right. \\
& \left.-\gamma_{2} \phi_{1}-\gamma_{3} \phi_{3}\right]-\frac{\partial \psi}{\partial r} \\
& u_{z}=\frac{\partial}{\partial z}\left[k_{1}\left(\phi_{01}-\gamma_{3} \phi_{1}\right)+k_{2}\left(\phi_{03}-\gamma_{2} \phi_{3}\right)\right. \\
& \left.+\gamma_{1}\left(k_{1} r \frac{\partial \phi_{1}}{\partial r}+k_{2} z \frac{\partial \phi_{3}}{\partial z}\right)\right]
\end{aligned}
$$

in which

$$
\begin{aligned}
& \nabla_{1}^{2} \phi_{01}+\nu_{1} \frac{\partial^{2} \phi_{01}}{\partial z^{2}}=0, \nabla_{1}^{2} \phi_{03}+\nu_{2} \frac{\partial^{2} \phi_{03}}{\partial z^{2}}=0 \\
& \nabla_{1}^{2} \phi_{1}+\nu_{2} \frac{\partial^{2} \phi_{1}}{\partial z^{2}}=0, \nabla_{1}^{2} \phi_{3}+\nu_{1} \frac{\partial^{2} \phi_{3}}{\partial z^{2}}=0 \cdots(2 \mathrm{c}, \mathrm{d}) \\
& \nabla_{1}^{2} \phi+\nu_{3} \frac{\partial^{2} \psi}{\partial z^{2}}=0, \nabla_{1}^{2} \equiv \frac{\partial^{2}}{\partial r^{2}}+\frac{1}{r} \frac{\partial}{\partial r}+\frac{1}{r^{2}} \frac{\partial^{2}}{\partial \theta^{2}} \\
& \gamma_{1}=\left\{\begin{array}{l}
1 \text { for } \nu_{1}=\nu_{2} \\
0 \text { for } \nu_{1} \neq \nu_{2}
\end{array}\right. \\
& \gamma_{2}=\left\{\begin{array}{l}
\frac{2 c_{11} \nu_{2}}{c_{11} \nu_{2}-c_{44}} \text { for } \nu_{1}=\nu_{2} \\
\frac{2 \nu_{2}}{\nu_{1}-\nu_{2}} \frac{c_{11} \nu_{1}-c_{44}}{c_{11} \nu_{2}-c_{44}} \text { for } \nu_{1} \neq \nu_{2}
\end{array}\right. \\
& r_{3}=\left\{\begin{array}{l}
0 \text { for } \nu_{1}=\nu_{2} \\
\frac{2 \nu_{2}}{\nu_{1}-\nu_{2}} \text { for } \nu_{1} \neq \nu_{2}
\end{array}\right. \\
& k_{1}=\frac{c_{11} \nu_{1}-c_{44}}{c_{13}+c_{44}}, k_{2}=\frac{c_{11} \nu_{2}-c_{44}}{c_{13}+c_{44}} \\
& \nu_{3}=\frac{c_{44}}{c_{66}}=\frac{2 c_{44}}{c_{11}-c_{12}}
\end{aligned}
$$

and $c_{i j}$ denotes the elastic constant of transversely isotropic solids, and $\nu_{1}$ and $\nu_{2}$ are the roots of

$$
c_{11} c_{44} \nu^{2}+\left[c_{13}\left(c_{13}+2 c_{44}\right)-c_{11} c_{33}\right] \nu+c_{33} c_{44}=0
$$

Determining the coordinate system of a short cylinder such as Fig.1 and regarding that the displacement and stress field is even in $z$, potential functions are obtained from Eqs.(2a-e) in the form

$$
\begin{aligned}
& \phi_{03}=\sum_{m=0}^{\infty} \sum_{s=1}^{\infty} A_{m s} \cos m \theta J_{m}\left(\alpha_{m s} r\right) \cosh \frac{\alpha_{m s} z}{\sqrt{\nu_{2}}} \\
& \phi_{3}=\sum_{m=0}^{\infty} \sum_{s=1}^{\infty} C_{m s} \cos m \theta J_{m}\left(\alpha_{m s} r\right) \cosh \frac{\alpha_{m s} z}{\sqrt{\nu_{1}}} \\
& \phi_{01}=\sum_{m=0}^{\infty} \sum_{n=1}^{\infty} D_{m m} \cos m \theta \cos \beta_{n} z I_{m}\left(\sqrt{\nu_{1}} \beta_{n} r\right) \\
& \phi_{1}=\sum_{m=0}^{\infty} \sum_{n=1}^{\infty} F_{m n} \cos m \theta \cos \beta_{n} z I_{m}\left(\sqrt{\nu_{2}} \beta_{n} r\right)
\end{aligned}
$$

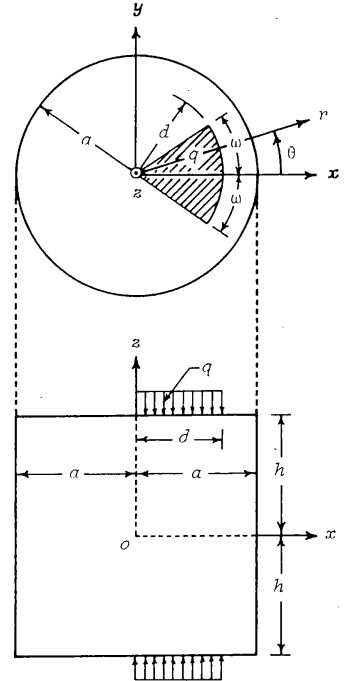

Fig.1 Coordinate system of short cylinder.

$$
\begin{aligned}
\psi= & \sum_{m=0}^{\infty} \sum_{s=1}^{\infty} B_{m s} \sin m \theta J_{m}\left(\alpha_{m s} r\right) \cosh \frac{\alpha_{m s} z}{\sqrt{\nu_{3}}} \\
& +\sum_{m=0}^{\infty} \sum_{n=1}^{\infty} E_{m n} \sin m \theta \cos \beta_{n} z I_{m}\left(\sqrt{\nu_{3}} \beta_{n} r\right)
\end{aligned}
$$

in which $A_{m s}, \cdots, E_{m n}$ are arbitrary constants to be determined from boundary conditions. Furthermore, $J_{m}\left(\alpha_{m s} r\right)$ and $I_{m}\left(\sqrt{\nu_{j}} \beta_{n} r\right)$ denote Bessel function and the modified Bessel function of the first kind, of order $m$, respectively, and

$$
\begin{aligned}
& \alpha_{m s}=\frac{\lambda_{m s}}{a}(m=0,1, \cdots, s=1,2, \cdots) \cdots \cdots \cdots \\
& \beta_{n}=\frac{n \pi}{h}(n=1,2, \cdots) \cdots \cdots \cdots \cdots \cdots \cdots \cdots \cdots \cdots
\end{aligned}
$$

in which $\lambda_{m s}$ is the root of a transcendental equation as stated later. Eqs. $(6 a, b)$ and a part of Eq. (6e) and Eqs. (6c, d) and another part of Eq. (6e) are the solutions satisfying the boundary conditions at the end faces and at the side surface, respectively.

In order to satisfy the boundary conditions as stated below, Fourier and Bessel expansions are needed. Since the expansions bring initial terms in Fourier or Bessel series, additional solutions are needed to treat the initial terms. They are as follows :

$$
\begin{aligned}
\phi_{01,0}= & \sum_{m=2}^{\infty} D_{m 0} \cos m \theta r^{m}-\frac{\nu_{1} \gamma_{4}}{\nu_{3}\left(\gamma_{2}-\gamma_{3}\right)} \\
& \cdot \sum_{m=1}^{\infty}\left(\gamma_{1} m-\gamma_{3}\right) F_{m 0} \cos m \theta\left[r^{m+2} / 2\right. \\
& \left.-(m+1) r^{m} z^{2} / \nu_{1}\right] \ldots \ldots \ldots \ldots \ldots \ldots
\end{aligned}
$$




$$
\begin{aligned}
\phi_{1,0}= & \frac{\nu_{2} \gamma_{4}}{\nu_{3}\left(\gamma_{2}-\gamma_{3}\right)} \sum_{m=1}^{\infty} F_{m 0} \cos m \theta\left[r^{m+2} / 2\right. \\
& \left.-(m+1) r^{m} z^{2} / \nu_{2}\right] \ldots \ldots \ldots \ldots \ldots \ldots \ldots(8 \mathrm{~b}) \\
\phi_{0}= & \gamma_{4} \sum_{m=1}^{\infty} F_{m 0} \sin m \theta\left[r^{m+2} / 2-(m+1) r^{m} z^{2} / \nu_{3}\right]
\end{aligned}
$$

in which

$$
\gamma_{4}=\frac{4 c_{11}}{c_{11}+c_{12}}
$$

and

$$
\begin{aligned}
& \phi_{3,00}=C_{00}\left(\frac{r^{2}}{2}-\frac{z^{2}}{\nu_{1}}\right) \\
& \phi_{1,00}=F_{00}\left(\frac{r^{2}}{2}-\frac{z^{2}}{\nu_{2}}\right)
\end{aligned}
$$

The generalized Hooke's law of transversely isotropic solids is

$$
\begin{aligned}
\sigma_{r r} & =c_{11} \varepsilon_{r r}+c_{12} \varepsilon_{\theta \theta}+c_{13} \varepsilon_{z z} \\
\sigma_{\theta \theta} & =c_{12} \varepsilon_{r r}+c_{11} \varepsilon_{\theta \theta}+c_{13} \varepsilon_{z z} \\
\sigma_{z z} & =c_{13} \varepsilon_{r r}+c_{13} \varepsilon_{\theta \theta}+c_{33} \varepsilon_{z z} \ldots \\
\sigma_{\theta z} & =2 c_{44} \varepsilon_{\theta z}, \sigma_{z r}=2 c_{44} \varepsilon_{z r} . . . \\
\sigma_{r \theta} & =\left(c_{11}-c_{12}\right) \varepsilon_{r \theta} \ldots \ldots \ldots \ldots
\end{aligned}
$$

in which $\sigma_{i j}$ and $\varepsilon_{i j}$ denote stress and strain components, respectively. If we substitute the potential functions of Eqs.(6a-e), (8a-c) and (10a, b) into Eqs.(1a-c), we obtain expressions for the displacement components. To make the explanation brief, we affix superscripts $(1),(2),(0,1)$ and $(0,0)$ successively to the displacement and stress components induced from Eqs. (6a, b, e), (6c, d, e), (8a-c) and (10a, b). Then, the displacement and stress components are expressed in the sum of four quantities as

$$
\begin{aligned}
& u_{r}=u_{r}^{(1)}+u_{r}^{(2)}+u_{r}^{(0,1)}+u_{r}^{(0,0)} \\
& \cdots \cdots \cdots \cdots \cdots \cdots \cdots \cdots \cdots \cdots \cdots \cdots \cdots \cdots \cdots \cdots \cdots \cdots \cdots \cdots \\
& \sigma_{r \theta}=\sigma_{r \theta}^{(1)}+\sigma_{r \theta}^{(2)}+\sigma_{r \theta}^{(0,1)}+\sigma_{r \theta}^{(0,0)}
\end{aligned}
$$

If we find the strain components from the displacement components obtained from Eqs.(1ac) and use Eqs.(11a-f), we obtain expressions for the stress components. For example, the expressions for $\sigma_{\theta z}$ and $\sigma_{z r}$ are

$$
\begin{aligned}
\sigma_{\theta z}^{(1)}= & -\frac{c_{44}}{2 \sqrt{\nu_{2}}} \sum_{m=0}^{\infty} \sum_{s=1}^{\infty} \alpha_{m s}^{2} \sin m \theta\left\langle\left(1+k_{2}\right)\right. \\
& \cdot\left[J_{m-1}\left(\alpha_{m s} r\right)+J_{m+1}\left(\alpha_{m s} r\right)\right] \\
& \cdot\left\{A_{m s} \sinh \frac{\alpha_{m s} z}{\sqrt{\nu_{2}}}\right. \\
& +C_{m s} \sqrt{\frac{\nu_{2}}{\nu_{1}}}\left[\gamma_{1} \frac{\alpha_{m s} z}{\sqrt{\nu_{1}}} \cosh \frac{\alpha_{m s} z}{\sqrt{\nu_{1}}}\right.
\end{aligned}
$$

$$
\begin{aligned}
& \left.\left.+\left(\gamma_{1}-\frac{k_{2} \gamma_{2}+\gamma_{3}}{1+k_{2}}\right) \sinh \frac{\alpha_{m s} z}{\sqrt{\nu_{1}}}\right]\right\} \\
& +\sqrt{\frac{\nu_{2}}{\nu_{3}}} B_{m s}\left[J_{m-1}\left(\alpha_{m s} r\right)\right. \\
& \left.\left.-J_{m+1}\left(\alpha_{m s} r\right)\right] \sinh \frac{\alpha_{m s} z}{\sqrt{\nu_{3}}}\right\rangle \cdots
\end{aligned}
$$$$
\sigma_{z r}^{(1)}=\frac{c_{44}}{2 \sqrt{\nu_{2}}} \sum_{m=0}^{\infty} \sum_{s=1}^{\infty} \alpha_{m s}^{2} \cos m \theta\left\langle\left(1+k_{2}\right)\right.
$$$$
\cdot\left[J_{m-1}\left(\alpha_{m s} r\right)-J_{m+1}\left(\alpha_{m s} r\right)\right]
$$$$
\cdot\left\{A_{m s} \sinh \frac{\alpha_{m s} z}{\sqrt{\nu_{2}}}+C_{m s} \sqrt{\frac{\nu_{2}}{\nu_{1}}}\left[\gamma_{1} \frac{\alpha_{m s} z}{\sqrt{\nu_{1}}}\right.\right.
$$$$
\left.\left.\cdot \cosh \frac{\alpha_{m s} z}{\sqrt{\nu_{1}}}+\left(\gamma_{1}-\frac{k_{2} \gamma_{2}+\gamma_{3}}{1+k_{2}}\right) \sinh \frac{\alpha_{m s} z}{\sqrt{\nu_{1}}}\right]\right\}
$$$$
+\sqrt{\frac{\nu_{2}}{\nu_{3}}} B_{m s}\left[J_{m-1}\left(\alpha_{m s} r\right)\right.
$$$$
\left.\left.+J_{m+1}\left(\alpha_{m s} r\right)\right] \sinh \frac{\alpha_{m s} z}{\sqrt{\nu_{3}}}\right\rangle
$$$$
\sigma_{\theta z}^{(2)}=\frac{c_{44} \sqrt{\nu_{1}}}{2} \sum_{m=0}^{\infty} \sum_{n=1}^{\infty} \beta_{n}^{2} \sin m \theta \sin \beta_{n} z
$$$$
\cdot\left\langleD _ { m n } ( 1 + k _ { 1 } ) \left[ I_{m-1}\left(\sqrt{\nu_{1}} \beta_{n} r\right)\right.\right.
$$$$
\left.-I_{m+1}\left(\sqrt{\nu_{1}} \beta_{n} r\right)\right]+F_{m n} \sqrt{\nu_{2} / \nu_{1}}
$$$$
\cdot\left\{\left[\gamma_{1} m\left(1+k_{1}\right)-\left(\gamma_{2}+k_{1} \gamma_{3}\right)\right]\right.
$$$$
\cdot I_{m-1}\left(\sqrt{\nu_{2}} \beta_{n} r\right)+\left[\gamma_{1} m\left(1+k_{1}\right)\right.
$$$$
\left.\left.+\gamma_{2}+k_{1} \gamma_{3}\right] I_{m+1}\left(\sqrt{\nu_{2}} \beta_{n} r\right)\right\}
$$$$
+E_{m n} \sqrt{\nu_{3} / \nu_{1}}\left[I_{m-1}\left(\sqrt{\nu_{3}} \beta_{n} r\right)\right.
$$$$
\left.\left.+I_{m+1}\left(\sqrt{\nu_{3}} \beta_{n} r\right)\right]\right\rangle
$$$$
\sigma_{z r}^{(2)}=-\frac{c_{44} \sqrt{\nu_{1}}}{2} \sum_{m=0}^{\infty} \sum_{n=1}^{\infty} \beta_{n}^{2} \cos m \theta \sin \beta_{n} z
$$$$
\cdot\left\langleD _ { m n } ( 1 + k _ { 1 } ) \left[ I_{m-1}\left(\sqrt{\nu_{1}} \beta_{n} r\right)\right.\right.
$$$$
\left.+I_{m+1}\left(\sqrt{\nu_{1}} \beta_{n} r\right)\right]+F_{m n} \sqrt{\nu_{2} / \nu_{1}}
$$$$
\text { - }\left\{\left[\gamma_{1} m\left(1+k_{1}\right)-\left(\gamma_{2}+k_{1} \gamma_{3}\right)\right] I_{m-1}\left(\sqrt{\nu_{2}} \beta_{n} r\right)\right.
$$$$
-\left[\gamma_{1} m\left(1+k_{1}\right)+\gamma_{2}+k_{1} \gamma_{3}\right] I_{m+1}\left(\sqrt{\nu_{2}} \beta_{n} r\right)
$$$$
\left.+2 \gamma_{1}\left(1+k_{1}\right) \sqrt{\nu_{2}} \beta_{n} r I_{m}\left(\sqrt{\nu_{2}} \beta_{n} r\right)\right\}
$$$$
+E_{m n} \sqrt{\nu_{3} / \nu_{1}}\left[I_{m-1}\left(\sqrt{\nu_{3}} \beta_{n} r\right)\right.
$$$$
\left.\left.-I_{m+1}\left(\sqrt{\nu_{3}} \beta_{n} r\right)\right]\right\rangle
$$$$
\sigma_{\theta z}^{(0,1)}=\sigma_{\theta z}^{(0,0)}=0
$$$$
\sigma_{z r}^{(0,1)}=\sigma_{z r}^{(0,0)}=0
$$

\section{BOUNDARY CONDITIONS}

We consider a short cylinder whose both end faces are subjected to uniformly distributed sectorial loads and whose side surface is free from surface tractions. 
The boundary conditions for that case become at $r=a$,

$$
\sigma_{r r}=0, \sigma_{r \theta}=0, \sigma_{r z}=0
$$

at $z= \pm h$,

$$
\sigma_{z r}=0, \sigma_{z \theta}=0, \sigma_{z z}=-p(r, \theta)
$$

in which

$$
p(r, \theta)=\left\{\begin{array}{l}
q \text { for } 0<r<d, 0 \leq \theta<\omega \text { and } \\
2 \pi-\omega<\theta \leq 2 \pi \cdots \ldots \ldots \ldots \\
0 \text { for the other domain }
\end{array}\right.
$$

From Eqs. (13b) and (14b), we obtain

$$
\left(\sigma_{\theta z}^{(2)}\right)_{z= \pm h}=\left(\sigma_{z r}^{(2)}\right)_{z= \pm h}=0
$$

Regarding Eq.(18) and imposing boundary conditions (16a, b) on Eqs.(14a) and (13a), we obtain the following relationships :

$$
B_{m s}=0, A_{m s}=a_{m s} \frac{\sinh \zeta_{1} h}{\sinh \zeta_{2} h} C_{m s}
$$

in which

$$
\begin{aligned}
& \zeta_{1}=\frac{\alpha_{m s}}{\sqrt{\nu_{1}}}, \zeta_{2}=\frac{\alpha_{m s}}{\sqrt{\nu_{2}}} \\
& a_{m s}=-\sqrt{\frac{\nu_{2}}{\nu_{1}}}\left[\gamma_{1}\left(1+\zeta_{1} h \operatorname{coth} \zeta_{1} h\right)-\frac{k_{2} \gamma_{2}+\gamma_{3}}{1+k_{2}}\right]
\end{aligned}
$$

Substituting relationships (19a, b) into Eq.(14a), the expression for $\sigma_{z r}^{(1)}$ is rewritten in the form

$$
\begin{aligned}
\sigma_{z r}^{(1)}= & c_{44} \frac{1+k_{2}}{\sqrt{\nu_{2}}} \sum_{m=0}^{\infty} \sum_{s=1}^{\infty} \alpha_{m s}^{2} C_{m s} \cos m \theta J_{m}^{\prime}\left(\alpha_{m s} r\right) \\
& \cdot\left\{a_{m s} \sinh \zeta_{1} h \frac{\sinh \zeta_{2} z}{\sinh \zeta_{2} h}+\sqrt{\frac{\nu_{2}}{\nu_{1}}}\left[\gamma_{1} \zeta_{1} z \cosh \zeta_{1} z\right.\right. \\
& \left.\left.+\left(\gamma_{1}-\frac{k_{2} \gamma_{2}+\gamma_{3}}{1+k_{2}}\right) \sinh \zeta_{1} z\right]\right\} \cdots \cdots \cdots \cdots(21)
\end{aligned}
$$

in which

$$
J_{m}^{\prime}\left(\alpha_{m s} r\right)=\frac{m J_{m}\left(\alpha_{m s} r\right)}{\alpha_{m s} r}-J_{m+1}\left(\alpha_{m s} r\right)
$$

Setting $r=a$ in Eq.(21) and taking $\lambda_{m s}$ as the positive root of the following transcendental equation:

$$
J_{m}^{\prime}\left(\alpha_{m s} a\right)=J_{m}^{\prime}\left(\lambda_{m s}\right)=0 \cdots
$$

we obtain

$$
\left(\sigma_{r z}^{(1)}\right)_{r=a}=0
$$

Regarding Eq.(24) and imposing boundary condition (15c) on Eq.(14b), we obtain the following relationship :

$$
\begin{aligned}
D_{m n}= & b_{m n}^{(1)} \frac{I_{m}\left(\sqrt{\nu_{2}} \beta_{n} a\right)}{I_{m}\left(\sqrt{\nu_{1}} \beta_{n} a\right)} F_{m n} \\
& +b_{m n}^{(2)} \frac{I_{m}\left(\sqrt{\nu_{3}} \beta_{n} a\right)}{I_{m}\left(\sqrt{\nu_{1}} \beta_{n} a\right)} E_{m n}
\end{aligned}
$$

in which

$$
b_{m n}^{(1)}=-\frac{1}{\Delta_{m n}}\left\{\gamma_{1}\left[m^{2}+\left(\sqrt{\nu_{2}} \beta_{n} a\right)^{2}\right]-\frac{\gamma_{2}+k_{1} \gamma_{3}}{1+k_{1}}\right.
$$

$$
\begin{array}{r}
\left.\cdot\left[m+\sqrt{\nu_{2}} \beta_{n} a \frac{I_{m+1}\left(\sqrt{\nu_{2}} \beta_{n} a\right)}{I_{m}\left(\sqrt{\nu_{2}} \beta_{n} a\right)}\right]\right\} \\
b_{m n}^{(2)}=-\frac{m}{\Delta_{m n}\left(1+k_{1}\right)} \cdots \ldots \ldots \ldots \ldots \ldots \ldots \ldots \ldots \ldots \ldots \ldots \ldots \ldots \ldots \\
\Delta_{m n}=m+\sqrt{\nu_{1}} \beta_{n} a \frac{I_{m+1}\left(\sqrt{\nu_{1}} \beta_{n} a\right)}{I_{m}\left(\sqrt{\nu_{1}} \beta_{n} a\right)} \ldots \ldots
\end{array}
$$

Thus, boundary conditions (15c) and (16a, b) were rigorously satisfied. By making use of relationships (19a, b) and (25), we can make the expressions for the displacement and stress components that eliminate arbitrary constants $B_{m s}, A_{m s}$ and $D_{m n}$ from the original expressions. For example, we have

$$
\begin{aligned}
\sigma_{r r}^{(1)}= & \sum_{m=0}^{\infty} \sum_{s=1}^{\infty} \alpha_{m s}^{2} C_{m s} \cos m \theta\left\langle\left(c_{11}-c_{12}\right) \frac{1}{\alpha_{m s} r}\right. \\
& \cdot\left[J_{m+1}\left(\alpha_{m s} r\right)+\frac{m(m-1)}{\alpha_{m s} r} J_{m}\left(\alpha_{m s} r\right)\right] \\
& \cdot\left[a_{m s} \sinh \zeta_{1} h \frac{\cosh \zeta_{2} z}{\sinh \zeta_{2} h}+\gamma_{1} \zeta_{1} z \sinh \zeta_{1} z\right. \\
& \left.-\gamma_{3} \cosh \zeta_{1} z\right]-J_{m}\left(\alpha_{m s} r\right) \\
& \cdot\left\{\left(c_{11}-c_{13} \frac{k_{2}}{\nu_{2}}\right) a_{m s} \sinh \zeta_{1} h \frac{\cosh \zeta_{2} z}{\sinh \zeta_{2} h}\right. \\
& +\gamma_{1}\left(c_{11}-c_{13} \frac{k_{2}}{\nu_{1}}\right) \zeta_{1} z \sinh \zeta_{1} z \\
& \left.\left.-\left[c_{11} \gamma_{3}+c_{13} \frac{k_{2}}{\nu_{1}}\left(2 \gamma_{1}-\gamma_{2}\right)\right] \cosh \zeta_{1} z\right\}\right\rangle
\end{aligned}
$$

$$
\begin{aligned}
\sigma_{z z}^{(1)}= & -\sum_{m=0}^{\infty} \sum_{s=1}^{\infty} \alpha_{m s}^{2} C_{m s} \cos m \theta J_{m}\left(\alpha_{m s} r\right) \\
& \cdot\left\{\left(c_{13}-c_{33} \frac{k_{2}}{\nu_{2}}\right) a_{m s} \sinh \zeta_{1} h \frac{\cosh \zeta_{2} z}{\sinh \zeta_{2} h}\right. \\
& +\gamma_{1}\left(c_{13}-c_{33} \frac{k_{2}}{\nu_{1}}\right) \zeta_{1} z \sinh \zeta_{1} z \\
& \left.-\left[c_{13} \gamma_{3}+c_{33} \frac{k_{2}}{\nu_{1}}\left(2 \gamma_{1}-\gamma_{2}\right)\right] \cosh \zeta_{1} z\right\}
\end{aligned}
$$

$$
\begin{aligned}
\sigma_{r \theta}^{(1)}= & -\left(c_{11}-c_{12}\right) \sum_{m=0}^{\infty} \sum_{s=1}^{\infty} \alpha_{m s}^{2} C_{m s} \sin m \theta \frac{m}{\alpha_{m s} r} \\
& \cdot\left[\frac{m-1}{\alpha_{m s} r} J_{m}\left(\alpha_{m s} r\right)-J_{m+1}\left(\alpha_{m s} r\right)\right] \\
& \cdot\left[a_{m s} \sinh \zeta_{1} h \frac{\cosh \zeta_{2} z}{\sinh \zeta_{2} h}+\gamma_{1} \zeta_{1} z \sinh \zeta_{1} z\right. \\
& \left.-\gamma_{3} \cosh \zeta_{1} z\right] \ldots \ldots \ldots \ldots \ldots \ldots \ldots \ldots \ldots \ldots \ldots \ldots \ldots \ldots \ldots \ldots \ldots \ldots
\end{aligned}
$$

$$
\begin{aligned}
\sigma_{r r}^{(2)}= & \nu_{1} \sum_{m=0}^{\infty} \sum_{n=1}^{\infty} \beta_{n}^{2} \cos m \theta \cos \beta_{n} z \\
& \cdot\left\langleF _ { m n } \left(b _ { m n } ^ { ( 1 ) } \frac { I _ { m } ( \sqrt { \nu _ { 2 } } \beta _ { n } a ) } { I _ { m } ( \sqrt { \nu _ { 1 } } \beta _ { n } a ) } \left\{ I_{m}\left(\sqrt{\nu_{1}} \beta_{n} r\right)\right.\right.\right. \\
\cdot & {\left[\left(c_{11}-c_{12}\right) \frac{m(m-1)}{\left(\sqrt{\nu_{1}} \beta_{n} r\right)^{2}}+c_{11}-c_{13} \frac{k_{1}}{\nu_{1}}\right] }
\end{aligned}
$$




$$
\begin{aligned}
& \left.-\left(c_{11}-c_{12}\right) \frac{I_{m+1}\left(\sqrt{\nu_{1}} \beta_{n} r\right)}{\sqrt{\nu_{1}} \beta_{n} r}\right\} \\
& +\frac{\nu_{2}}{\nu_{1}}\left\{I _ { m } ( \sqrt { \nu _ { 2 } } \beta _ { n } r ) \left[\left(c_{11}-c_{12}\right)\right.\right. \\
& \cdot\left(\gamma_{1} m-\gamma_{2}\right) \frac{m(m-1)}{\left(\sqrt{\nu_{2}} \beta_{n} r\right)^{2}}+\gamma_{1}\left(c_{11}(m+1)\right. \\
& \left.\left.+c_{12}-c_{13} \frac{k_{1}}{\nu_{2}} m\right)-c_{11} \gamma_{2}+c_{13} \frac{k_{1}}{\nu_{2}} \gamma_{3}\right] \\
& +I_{m+1}\left(\sqrt{\nu_{2}} \beta_{n} r\right)\left[\left(c_{11}-c_{12}\right) \frac{\gamma_{1} m^{2}+\gamma_{2}}{\sqrt{\nu_{2}} \beta_{n} r}\right. \\
& \left.\left.\left.+\gamma_{1}\left(c_{11}-c_{13} \frac{k_{1}}{\nu_{2}}\right) \sqrt{\nu_{2}} \beta_{n} r\right]\right\}\right) \\
& +E_{m n}\left(b_{m n}^{(2)} \frac{I_{m}\left(\sqrt{\nu_{3}} \beta_{n} a\right)}{I_{m}\left(\sqrt{\nu_{1}} \beta_{n} a\right)}\right. \\
& \cdot\left\{I _ { m } ( \sqrt { \nu _ { 1 } } \beta _ { n } r ) \left[\left(c_{11}-c_{12}\right) \frac{m(m-1)}{\left(\sqrt{\nu_{1}} \beta_{n} r\right)^{2}}\right.\right. \\
& \left.\left.+c_{11}-c_{13} \frac{k_{1}}{\nu_{1}}\right]-\left(c_{11}-c_{12}\right) \frac{I_{m+1}\left(\sqrt{\nu_{1}} \beta_{n} r\right)}{\sqrt{\nu_{1}} \beta_{n} r}\right\} \\
& +\frac{\nu_{3}}{\nu_{1}}\left(c_{11}-c_{12}\right) \frac{m}{\sqrt{\nu_{3}} \beta_{n} r}\left[\frac{m-1}{\sqrt{\nu_{3}} \beta_{n} r} I_{m}\left(\sqrt{\nu_{3}} \beta_{n} r\right)\right. \\
& \left.\left.\left.+I_{m+1}\left(\sqrt{\nu_{3}} \beta_{n} r\right)\right]\right)\right\rangle \ldots \ldots \ldots \ldots \ldots \ldots(27 \mathrm{~b})
\end{aligned}
$$

$$
\sigma_{z z}^{(2)}=\nu_{1} \sum_{m=0}^{\infty} \sum_{n=1}^{\infty} \beta_{n}^{2} \cos m \theta \cos \beta_{n} z
$$$$
\cdot\left\langleF _ { m n } \left( b_{m n}^{(1)} \frac{I_{m}\left(\sqrt{\nu_{2}} \beta_{n} a\right)}{I_{m}\left(\sqrt{\nu_{1}} \beta_{n} a\right)}\right.\right.
$$$$
\cdot\left(c_{13}-c_{33} \frac{k_{1}}{\nu_{1}}\right) I_{m}\left(\sqrt{\nu_{1}} \beta_{n} r\right)+\frac{\nu_{2}}{\nu_{1}}\left\{\left(\gamma _ { 1 } \left[c_{13}(m+2)\right.\right.\right.
$$$$
\left.\left.-c_{33} \frac{k_{1}}{\nu_{2}} m\right]-c_{13} \gamma_{2}+c_{33} \frac{k_{1}}{\nu_{2}} \gamma_{3}\right) I_{m}\left(\sqrt{\nu_{2}} \beta_{n} r\right)
$$$$
\left.\left.+\gamma_{1}\left(c_{13}-c_{33} \frac{k_{1}}{\nu_{2}}\right) \sqrt{\nu_{2}} \beta_{n} r I_{m+1}\left(\sqrt{\nu_{2}} \beta_{n} r\right)\right\}\right)
$$$$
+E_{m n} b_{m n}^{(2)}\left(c_{13}-c_{33} \frac{k_{1}}{\nu_{1}}\right) \frac{I_{m}\left(\sqrt{\nu_{3}} \beta_{n} a\right)}{I_{m}\left(\sqrt{\nu_{1}} \beta_{n} a\right)}
$$$$
\left.\cdot I_{m}\left(\sqrt{\nu_{1}} \beta_{n} r\right)\right\rangle
$$

$\sigma_{r \theta}^{(2)}=-\left(c_{11}-c_{12}\right) \nu_{1} \sum_{m=0}^{\infty} \sum_{n=1}^{\infty} \beta_{n}^{2} \sin m \theta \cos \beta_{n} z\left\langle m F_{m n}\right.$

$$
\begin{aligned}
& \cdot\left(b_{m n}^{(1)} \frac{I_{m}\left(\sqrt{\nu_{2}} \beta_{n} a\right)}{I_{m}\left(\sqrt{\nu_{1}} \beta_{n} a\right)} \frac{1}{\sqrt{\nu_{1}} \beta_{n} r}\right. \\
& \cdot\left[\frac{m-1}{\sqrt{\nu_{1}} \beta_{n} r} I_{m}\left(\sqrt{\nu_{1}} \beta_{n} r\right)+I_{m+1}\left(\sqrt{\nu_{1}} \beta_{n} r\right)\right] \\
& +\frac{\nu_{2}}{\nu_{1}}\left\{I _ { m } ( \sqrt { \nu _ { 2 } } \beta _ { n } r ) \left[\gamma_{1}+\left(\gamma_{1} m-\gamma_{2}\right)\right.\right. \\
& \left.\left.\left.\cdot \frac{m-1}{\left(\sqrt{\nu_{2}} \beta_{n} r\right)^{2}}\right]-\left(\gamma_{1}+\gamma_{2}\right) \frac{I_{m+1}\left(\sqrt{\nu_{2}} \beta_{n} r\right)}{\sqrt{\nu_{2}} \beta_{n} r}\right\}\right)
\end{aligned}
$$

$$
\begin{aligned}
& +E_{m n}\left(\dot { b _ { m n } ^ { ( 2 ) } } \frac { I _ { m } ( \sqrt { \nu _ { 3 } } \beta _ { n } a ) } { I _ { m } ( \sqrt { \nu _ { 1 } } \beta _ { n } a ) } \frac { m } { \sqrt { \nu _ { 1 } } \beta _ { n } r } \left[\frac{m-1}{\sqrt{\nu_{1}} \beta_{n} r}\right.\right. \\
& \left.\cdot I_{m}\left(\sqrt{\nu_{1}} \beta_{n} r\right)+I_{m+1}\left(\sqrt{\nu_{1}} \beta_{n} r\right)\right] \\
& +\frac{\nu_{3}}{\nu_{1}}\left\{I_{m}\left(\sqrt{\nu_{3}} \beta_{n} r\right)\left[\frac{1}{2}+\frac{m(m-1)}{\left(\sqrt{\nu_{3}} \beta_{n} r\right)^{2}}\right]\right. \\
& \left.\left.\left.-\frac{I_{m+1}\left(\sqrt{\nu_{3}} \beta_{n} r\right)}{\sqrt{\nu_{3}} \beta_{n} r}\right\}\right)\right\rangle \ldots \ldots \ldots \ldots \ldots \ldots(29 \mathrm{~b})
\end{aligned}
$$

and the expressions for the stress components induced from additional solutions (8a-c) and (10a, b) are

$$
\begin{aligned}
& \sigma_{r r}^{(0,1)}=\left(c_{11}-c_{12}\right) \sum_{m=2}^{\infty} D_{m 0} m(m-1) \cos m \theta r^{m-2} \\
& +\sum_{m=1}^{\infty} F_{m 0}(m+1)\left[m\left(c_{11}-c_{12}\right)\right. \\
& \left.+\left(c_{11}+c_{12}\right)\left(2-\gamma_{4}\right)\right] \cos m \theta r^{m} \\
& \sigma_{z z}^{(0,1)}=2 c_{13}\left(2-\gamma_{4}\right) \sum_{m=1}^{\infty} F_{m 0}(m+1) \cos m \theta r^{m} \\
& \sigma_{r \theta}^{(0,1)}=-\left(c_{11}-c_{12}\right)\left[\sum_{m=1}^{\infty} D_{m 0} m(m-1) \sin m \theta r^{m-2}\right. \\
& \left.+\sum_{m=1}^{\infty} F_{m 0} m(m+1) \sin m \theta r^{m}\right] \\
& \sigma_{r r}^{(0,0)}=F_{00}\left[\left(c_{11}+c_{12}\right)\left(2 \gamma_{1}-\gamma_{2}\right)+\frac{2 k_{1}}{\nu_{2}} c_{13} \gamma_{3}\right]-C_{00} \\
& \cdot\left[\gamma_{3}\left(c_{11}+c_{12}\right)+\frac{2 k_{2}}{\nu_{1}} c_{13}\left(2 \gamma_{1}-\gamma_{2}\right)\right] \cdots \\
& \sigma_{z z}^{(0,0)}=2 F_{00}\left[c_{13}\left(2 \gamma_{1}-\gamma_{2}\right)+c_{33} \frac{k_{1} \gamma_{3}}{\nu_{2}}\right]-2 C_{00} \\
& \cdot\left[c_{13} \gamma_{3}+c_{33} \frac{k_{2}}{\nu_{1}}\left(2 \gamma_{1}-\gamma_{2}\right)\right] \text {. }
\end{aligned}
$$

\section{SYSTEMS OF LINEAR ALGE- BRAIC EQUATIONS}

In this article, we consider satisfying boundary conditions $(15 \mathrm{a}, \mathrm{b})$ and $(16 \mathrm{c})$. The Fourier-Bessel expansion of load function (17), under the condition of Eq.(23), is as follows :

$$
\begin{aligned}
p(r, \theta)= & e_{00}+\sum_{s=1}^{\infty} e_{0 s} J_{0}\left(\alpha_{0 s} r\right)+\sum_{m=1}^{\infty} \sum_{s=1}^{\infty} e_{m s} \cos m \theta \\
& \cdot J_{m}\left(\alpha_{m s} r\right) \ldots \ldots \ldots \ldots \ldots \ldots \ldots \ldots \ldots \ldots \ldots \ldots \ldots \ldots \ldots \ldots \ldots
\end{aligned}
$$

in which

$$
\begin{aligned}
& e_{00}=\frac{q \omega}{\pi}\left(\frac{d}{a}\right)^{2} \ldots \ldots \ldots \ldots \ldots . . . . . . \\
& e_{0 s}=\frac{2 q \omega}{\pi}\left(\frac{d}{a}\right) \frac{J_{1}\left(\lambda_{0 s} \frac{d}{a}\right)}{\lambda_{0 s} J_{0}^{2}\left(\lambda_{0 s}\right)}
\end{aligned}
$$




$$
\begin{aligned}
e_{m s}= & \frac{4 q \sin m \omega}{m \pi\left(\lambda_{m s}^{2}-m^{2}\right) J_{m}^{2}\left(\lambda_{m s}\right)}\left[\lambda_{m s}\left(\frac{d}{a}\right) J_{m+1}\left(\lambda_{m s} \frac{d}{a}\right)\right. \\
& \left.+2 m \sum_{k=0}^{\infty} J_{m+2+2 k}\left(\lambda_{m s} \frac{d}{a}\right)\right] \ldots \ldots \ldots \ldots \ldots \ldots(31 \mathrm{c})
\end{aligned}
$$

To satisfy boundary conditions $(15 \mathrm{a}, \mathrm{b})$, we have to expand $\sigma_{r r}^{(1)}$ and $\sigma_{r \theta}^{(1)}$ at $r=a$ of Eqs. (27a) and (29a) into Fourier series. Then, we obtain

$$
\begin{aligned}
\left(\sigma_{r r}^{(1)}\right)_{r=a}= & \sum_{m=0}^{\infty} \sum_{s=1}^{\infty} \frac{C_{m s}}{a^{2}} \sinh \zeta_{1} h J_{m}\left(\lambda_{m s}\right) \cos m \theta \\
& \cdot\left({ }_{s} t_{m 0}^{(1)}+\sum_{n=1}^{\infty}{ }_{s} t_{m n}^{(1)} \cos \beta_{n} z\right) \ldots \ldots \ldots \ldots(32) \\
\left(\sigma_{r \theta}^{(1)}\right)_{r=a}= & \left(c_{11}-c_{12}\right) \sum_{m=0}^{\infty} \sum_{s=1}^{\infty} \frac{m C_{m s}}{a^{2}} \sinh \zeta_{1} h J_{m}\left(\lambda_{m s}\right) \\
& \cdot \sin m \theta\left({ }_{s} t_{m 0}^{(2)}+\sum_{n=1}^{\infty}{ }_{s} t_{m n}^{(2)} \cos \beta_{n} z\right) \cdots \cdots(33)
\end{aligned}
$$

in which ${ }_{s} t_{m 0}^{(1)}, \cdots,{ }_{s} t_{m n}^{(2)}$ are Fourier coefficients and ${ }_{s} t_{00}^{(1)}=0$.

Using Eq.(32) and imposing boundary condition (15a) on Eqs.(27b-d), we obtain three systems of linear algebraic equations in the form

$$
\begin{aligned}
& \sum_{s=1}^{\infty} \frac{C_{m s}}{a^{2}} \sinh \zeta_{1} h_{s} t_{m n}^{(1)} J_{m}\left(\lambda_{m s}\right)+\beta_{n}^{2} F_{m n} I_{m}\left(\sqrt{\nu_{2}} \beta_{n} a\right) \\
& \cdot\left\langleb _ { m n } ^ { ( 1 ) } \nu _ { 1 } \left[\left(c_{11}-c_{12}\right) \frac{m(m-1)}{\left(\sqrt{\nu_{1}} \beta_{n} a\right)^{2}}\right.\right. \\
& \left.+c_{11}-c_{13} \frac{k_{1}}{\nu_{1}}-\frac{c_{11}-c_{12}}{\sqrt{\nu_{1}} \beta_{n} a} \frac{I_{m+1}\left(\sqrt{\nu_{1}} \beta_{n} a\right)}{I_{m}\left(\sqrt{\nu_{1}} \beta_{n} a\right)}\right] \\
& +\nu_{2}\left\{\left(c_{11}-c_{12}\right)\left(\gamma_{1} m-\gamma_{2}\right) \frac{m(m-1)}{\left(\sqrt{\nu_{2}} \beta_{n} a\right)^{2}}\right. \\
& +\gamma_{1}\left[c_{11}(m+1)+c_{12}-c_{13} \frac{k_{1}}{\nu_{2}} m\right] \\
& -c_{11} \gamma_{2}+c_{13} \frac{k_{1} \gamma_{3}}{\nu_{2}}+\frac{I_{m+1}\left(\sqrt{\nu_{2}} \beta_{n} a\right)}{I_{m}\left(\sqrt{\nu_{2}} \beta_{n} a\right)} \\
& \cdot\left[\left(c_{11}-c_{12}\right) \frac{\gamma_{1} m^{2}+\gamma_{2}}{\sqrt{\nu_{2}} \beta_{n} a}+\gamma_{1}\left(c_{11}-c_{13} \frac{k_{1}}{\nu_{2}}\right)\right. \\
& \left.\left.\left.\cdot \sqrt{\nu_{2}} \beta_{n} a\right]\right\}\right\rangle+\beta_{n}^{2} E_{m n} I_{m}\left(\sqrt{\nu_{3}} \beta_{n} a\right) \\
& \cdot\left\{b _ { m n } ^ { ( 2 ) } \nu _ { 1 } \left[\left(c_{11}-c_{12}\right) \frac{m(m-1)}{\left(\sqrt{\nu_{1}} \beta_{n} a\right)^{2}}+c_{11}-c_{13} \frac{k_{1}}{\nu_{1}}\right.\right. \\
& \left.-\frac{c_{11}-c_{12}}{\sqrt{\nu_{1}} \beta_{n} a} \frac{I_{m+1}\left(\sqrt{\nu_{1}} \beta_{n} a\right)}{I_{m}\left(\sqrt{\nu_{1}} \beta_{n} a\right)}\right] \\
& +\nu_{3}\left(c_{11}-c_{12}\right) \frac{m}{\sqrt{\nu_{3}} \beta_{n} a}\left[\frac{m-1}{\sqrt{\nu_{3}} \beta_{n} a}\right. \\
& \left.\left.+\frac{I_{m+1}\left(\sqrt{\nu_{3}} \beta_{n} a\right)}{I_{m}\left(\sqrt{\nu_{3}} \beta_{n} a\right)}\right]\right\}=0,(m \geq 0)
\end{aligned}
$$

$\sum_{s=1}^{\infty} \frac{C_{m s}}{a^{2}} \sinh \zeta_{1} h_{s} t_{m 0}^{(1)} J_{m}\left(\lambda_{m s}\right)+\delta_{m}^{\prime} D_{m 0} a^{m-2}$

$\cdot\left(c_{11}-c_{12}\right) m(m-1)+F_{m 0} a^{m}(m+1)\left[m\left(c_{11}-c_{12}\right)\right.$

$$
\left.+\left(c_{11}+c_{12}\right)\left(2-r_{4}\right)\right]=0,(m \geq 1)
$$

in which

$$
\begin{aligned}
& \delta_{m}^{\prime}=\left\{\begin{array}{l}
0 \text { for } m=1 \\
1 \text { for } m \geq 2
\end{array}\right. \\
& F_{00}\left[\left(c_{11}+c_{12}\right)\left(2 \gamma_{1}-\gamma_{2}\right)+\frac{2 k_{1}}{\nu_{2}} c_{13} \gamma_{3}\right] \\
& -C_{00}\left[\gamma_{3}\left(c_{11}+c_{12}\right)+\frac{2 k_{2}}{\nu_{1}} c_{13}\left(2 \gamma_{1}-\gamma_{2}\right)\right]=0
\end{aligned}
$$

Using Eq.(33) and imposing boundary condition (15b) on Eqs.(29b,c), we obtain two systems of linear algebraic equations in the form

$$
\begin{aligned}
& \sum_{s=1}^{\infty} \frac{m C_{m s}}{a^{2}} \sinh \zeta_{1} h_{s} t_{m n}^{(2)} J_{m}\left(\lambda_{m s}\right)-\beta_{n}^{2} m F_{m n} I_{m}\left(\sqrt{\nu_{2}} \beta_{n} a\right) \\
& \cdot\left\{\frac{b_{m n}^{(1)} \nu_{1}}{\sqrt{\nu_{1}} \beta_{n} a}\left[\frac{m-1}{\sqrt{\nu_{1}} \beta_{n} a}+\frac{I_{m+1}\left(\sqrt{\nu_{1}} \beta_{n} a\right)}{I_{m}\left(\sqrt{\nu_{1}} \beta_{n} a\right)}\right]\right. \\
& +\nu_{2}\left[\gamma_{1}+\left(\gamma_{1} m-\gamma_{2}\right) \frac{m-1}{\left(\sqrt{\nu_{2}} \beta_{n} a\right)^{2}}\right. \\
& \left.\left.-\frac{\gamma_{1}+\gamma_{2}}{\sqrt{\nu_{2}} \beta_{n} a} \frac{I_{m+1}\left(\sqrt{\nu_{2}} \beta_{n} a\right)}{I_{m}\left(\sqrt{\nu_{2}} \beta_{n} a\right)}\right]\right\}-\beta_{n}^{2} E_{m n} I_{m}\left(\sqrt{\nu_{3}} \beta_{n} a\right) \\
& \cdot\left\{b_{m n}^{(2)} \frac{\nu_{1} m}{\sqrt{\nu_{1}} \beta_{n} a}\left[\frac{m-1}{\sqrt{\nu_{1}} \beta_{n} a}+\frac{I_{m+1}\left(\sqrt{\nu_{1}} \beta_{n} a\right)}{I_{m}\left(\sqrt{\nu_{1}} \beta_{n} a\right)}\right]+\nu_{3}\left[\frac{1}{2}\right.\right. \\
& \left.\left.+\frac{m(m-1)}{\left(\sqrt{\nu_{3}} \beta_{n} a\right)^{2}}-\frac{1}{\sqrt{\nu_{3}} \beta_{n} a} \frac{I_{m+1}\left(\sqrt{\nu_{3}} \beta_{n} a\right)}{I_{m}\left(\sqrt{\nu_{3}} \beta_{n} a\right)}\right]\right\}=0 \text {, } \\
& (m \geq 0) \\
& \sum_{s=1}^{\infty} \frac{m C_{m s}}{a^{2}} \sinh \zeta_{1} h_{s} t_{m 0}^{(2)} J_{m}\left(\lambda_{m s}\right)-\delta_{m}^{\prime} D_{m 0} a^{m-2} m(m-1) \\
& -F_{m 0} a^{m} m(m+1)=0,(m \geq 1)
\end{aligned}
$$

To satisfy boundary condition (16c), we have to expand $\sigma_{z z}^{(2)}$ at $z= \pm h$ of Eq. (28b) and $\sigma_{z z}^{(0,1)}$ of Eq.(28c) into Bessel series, under Eq.(23). Then, we obtain

$$
\begin{aligned}
& \left(\sigma_{z z}^{(2)}\right)_{z= \pm h}=\sum_{m=0}^{\infty} \sum_{n=1}^{\infty}(-1)^{n} \beta_{n}^{2} \cos m \theta\left\{F_{m n} I_{m}\left(\sqrt{\nu_{2}} \beta_{n} a\right)\right. \\
& \cdot\left[{ }_{n} t_{00}^{(3)}+\sum_{s=1}^{\infty}{ }_{n} t_{m s}^{(3)} J_{m}\left(\alpha_{m s} r\right)\right]+E_{m n} \\
& \left.\cdot I_{m}\left(\sqrt{\nu_{3}} \beta_{n} a\right)\left[{ }_{n} t_{00}^{(4)}+\sum_{s=1}^{\infty}{ }_{n} t_{m s}^{(4)} J_{m}\left(\alpha_{m s} r\right)\right]\right\} \\
& \sigma_{z z}^{(0,1)}=2 c_{13}\left(2-\gamma_{4}\right) \sum_{m=1}^{\infty} F_{m 0} a^{m}(m+1) \cos m \theta \\
& \cdot \sum_{s=1}^{\infty} t_{m s}^{(5)} J_{m}\left(\alpha_{m s} r\right)
\end{aligned}
$$

in which ${ }_{n} t_{00}^{(3)}={ }_{n} t_{00}^{(4)}=0, \cdots, t_{m s}^{(5)}$ are Fourier coefficients.

Using Eqs.(30) and (37a, b) and imposing boundary condition (16c) on Eqs.(28a,d), we obtain two systems of linear algebraic equations in the form 


$$
\begin{aligned}
& \frac{C_{m s}}{a^{2}} \lambda_{m s}^{2} \sinh \zeta_{1} h\left\{\left(c_{13}-c_{33} \frac{k_{2}}{\nu_{2}}\right) a_{m s} \operatorname{coth} \zeta_{2} h+\gamma_{1}\right. \\
& \cdot\left(c_{13}-c_{33} \frac{k_{2}}{\nu_{1}}\right) \zeta_{1} h-\left[c_{13} \gamma_{3}+c_{33} \frac{k_{2}}{\nu_{1}}\left(2 \gamma_{1}-\gamma_{2}\right)\right] \\
& \left.\cdot \operatorname{coth} \zeta_{1} h\right\}-\sum_{n=1}^{\infty}(-1)^{n} \beta_{n}^{2}\left[F_{m n} I_{m}\left(\sqrt{\nu_{2}} \beta_{n} a\right)_{n} t_{m s}^{(3)}\right. \\
& \left.+E_{m n} I_{m}\left(\sqrt{\nu_{3}} \beta_{n} a\right)_{n} t_{m s}^{(4)}\right]-2 c_{13}\left(2-\gamma_{4}\right) \delta_{m}^{*} F_{m 0} a^{m} \\
& \cdot(m+1) t_{m s}^{(5)}=e_{m s},(m \geq 0) \\
& 2 F_{00}\left[c_{13}\left(2 \gamma_{1}-\gamma_{2}\right)+c_{33} \frac{k_{1} \gamma_{3}}{\nu_{2}}\right]-2 C_{00}\left[c_{13} \gamma_{3}+c_{33}\right. \\
& \left.\frac{k_{2}}{\nu_{1}}\left(2 \gamma_{1}-\gamma_{2}\right)\right]=-e_{00}
\end{aligned}
$$

in which

$$
\begin{aligned}
& \delta_{m}^{*}=\left\{\begin{array}{l}
0 \text { for } m=0 \\
1 \text { for } m \geq 1
\end{array}\right. \\
& { }_{n} t_{m s}^{(3)}=\frac{2 \lambda_{m s}^{2}}{J_{m}\left(\lambda_{m s}\right)\left(\lambda_{m s}^{2}-m^{2}\right)}\left\langle b_{m n}^{(1)} \nu_{1}\left(c_{13}-c_{33} \frac{k_{1}}{\nu_{1}}\right)\right. \\
& \cdot \frac{1}{\lambda_{m s}^{2}+\left(\sqrt{\nu_{1}} \beta_{n} a\right)^{2}}\left[m+\sqrt{\nu_{1}} \beta_{n} a \frac{I_{m+1}\left(\sqrt{\nu_{1}} \beta_{n} a\right)}{I_{m}\left(\sqrt{\nu_{1}} \beta_{n} a\right)}\right] \\
& +\frac{\nu_{2}}{\lambda_{m s}^{2}+\left(\sqrt{\nu_{2}} \beta_{n} a\right)^{2}}\left(\left\{\gamma_{1}\left[c_{13}(m+2)-c_{33} \frac{k_{1}}{\nu_{2}} m\right]\right.\right. \\
& \left.-c_{13} \gamma_{2}+c_{33} \frac{k_{1}}{\nu_{2}} \gamma_{3}\right\}\left[m+\sqrt{\nu_{2}} \beta_{n} a \frac{I_{m+1}\left(\sqrt{\nu_{2}} \beta_{n} a\right)}{I_{m}\left(\sqrt{\nu_{2}} \beta_{n} a\right)}\right] \\
& +\gamma_{1}\left(c_{13}-c_{33} \frac{k_{1}}{\nu_{2}}\right) \sqrt{\nu_{2}} \beta_{n} a\left\{\sqrt{\nu_{2}} \beta_{n} a-(m+2)\right. \\
& \cdot \frac{I_{m+1}\left(\sqrt{\nu_{2}} \beta_{n} a\right)}{I_{m}\left(\sqrt{\nu_{2}} \beta_{n} a\right)}-\frac{2}{\lambda_{m s}^{2}+\left(\sqrt{\nu_{2}} \beta_{n} a\right)^{2}}\left[m \sqrt{\nu_{2}} \beta_{n} a\right. \\
& \left.\left.\left.\left.-\lambda_{m s}^{2} \frac{I_{m+1}\left(\sqrt{\nu_{2}} \beta_{n} a\right)}{I_{m}\left(\sqrt{\nu_{2}} \beta_{n} a\right)}\right]\right\}\right)\right\rangle \text {. } \\
& { }_{n} t_{m s}^{(4)}=\frac{2 \lambda_{m s}^{2}}{J_{m}\left(\lambda_{m s}\right)\left(\lambda_{m s}^{2}-m^{2}\right)} b_{m n}^{(2)} \nu_{1}\left(c_{13}-c_{33} \frac{k_{1}}{\nu_{1}}\right) \\
& \cdot \frac{1}{\lambda_{m s}^{2}+\left(\sqrt{\nu_{1}} \beta_{n} a\right)^{2}}\left[m+\sqrt{\nu_{1}} \beta_{n} a \frac{I_{m+1}\left(\sqrt{\nu_{1}} \beta_{n} a\right)}{I_{m}\left(\sqrt{\nu_{1}} \beta_{n} a\right)}\right] \\
& t_{m s}^{(5)}=\frac{2 m}{J_{m}\left(\lambda_{m s}\right)\left(\lambda_{m s}^{2}-m^{2}\right)}
\end{aligned}
$$

Solving the system of Eqs.(34b) and (36b) with $D_{m 0}$ and $F_{m 0}$, we obtain

$$
\begin{aligned}
& D_{m 0} a^{m-2}=\frac{1}{\Delta_{1}(m-1)} \sum_{s=1}^{\infty} \frac{C_{m s}}{a^{2}} \sinh \zeta_{1} h J_{m}\left(\lambda_{m s}\right)\left\{t_{m 0}^{(1)}\right. \\
& \left.+\left[m\left(c_{11}-c_{12}\right)+\left(c_{11}+c_{12}\right)\left(2-\gamma_{4}\right)\right]_{s} t_{m 0}^{(2)}\right\}, \\
& (m \geq 2) \text {. } \\
& F_{m 0} a^{m}=-\frac{1}{\Delta_{1}(m+1)} \sum_{s=1}^{\infty} \frac{C_{m s}}{a^{2}} \sinh \zeta_{1} h J_{m}\left(\lambda_{m s}\right)\left[s t_{m 0}^{(1)}\right. \\
& \left.+m\left(c_{11}-c_{12}\right)_{s} t_{m 0}^{(2)}\right],(m \geq 1) \cdots \cdots(40 \mathrm{~b})
\end{aligned}
$$

in which

$$
\Delta_{1}=\left(c_{11}+c_{12}\right)\left(2-\gamma_{4}\right)
$$

Solving the system of Eqs.(34c) and (38b) with $C_{00}$ and $F_{00}$, we obtain

$$
\begin{aligned}
& C_{00}=\frac{e_{00}}{2 \Delta_{2}}\left[\left(c_{11}+c_{12}\right)\left(2 \gamma_{1}-\gamma_{2}\right)+\frac{2 k_{1}}{\nu_{2}} c_{13} \gamma_{3}\right] \\
& F_{00}=\frac{e_{00}}{2 \Delta_{2}}\left[\gamma_{3}\left(c_{11}+c_{12}\right)+\frac{2 k_{2}}{\nu_{1}} c_{13}\left(2 \gamma_{1}-\gamma_{2}\right)\right]
\end{aligned}
$$

in which

$$
\begin{aligned}
\Delta_{2}= & {\left[2 c_{13}^{2}-c_{33}\left(c_{11}+c_{12}\right)\right] } \\
& \cdot\left[\frac{k_{1} \gamma_{3}^{2}}{\nu_{2}}-\frac{k_{2}}{\nu_{1}}\left(2 \gamma_{1}-\gamma_{2}\right)^{2}\right]
\end{aligned}
$$

The system of Eqs.(34a), (36a), (38a) and (40b) with $C_{m s}, F_{m n}, E_{m n}$ and $F_{m 0}$ can be numerically solved by an iterative method. Once all the arbitrary constants are determined, the values of the displacement and stress components of the short cylinder are completely determined. In order to facilitate numerical calculations of the system of linear algebraic equations, displacements and stresses, it is convenient to replace the arbitrary constants previously used with the following ones :

$$
\begin{aligned}
& \frac{c_{44} C_{m s}}{q a^{2}} \sinh \zeta_{1} h=\bar{C}_{m s} ; \frac{c_{44} F_{m n}}{q a^{2}} I_{m}\left(\sqrt{\nu_{2}} \beta_{n} a\right)=\bar{F}_{m n} ; \\
& \frac{c_{44} E_{m n}}{q a^{2}} I_{m}\left(\sqrt{\nu_{3}} \beta_{n} a\right)=\bar{E}_{m n} ; \frac{c_{44} D_{m 0} a^{m-2}}{q}=\bar{D}_{m 0} ; \\
& \frac{c_{44} F_{m 0} a^{m}}{q}=\bar{F}_{m 0} ; \frac{c_{44} F_{00}}{q}=\bar{F}_{00} ; \frac{c_{44} C_{00}}{q}=\bar{C}_{00} \cdots . .(44)
\end{aligned}
$$

\section{NUMERICAL RESULTS}

Numerical calculations were made for transversely isotropic and isotropic, short cylinders with $h / a=1.0, d / a=0.3$ and $\omega=0.3 \pi$. Magnesium and cadmium crystals, as examples of transversely isotropic materials, and an isotropic material with Poisson's ratio $\nu=0.25$ were treated. The values of the elastic constants of these materials, as determined by Huntington ${ }^{8)}$, are given in Table 1 . The roots of the transcendental equation were calculated by the Regula-Falsi method. Numerical results were obtained by taking the first 40 terms for $m$ and 38 terms for $s$ and $n$ in the series. The check for the convergence of $\sigma_{r r}, \sigma_{\theta \theta}$ and $\sigma_{z z}$ at $\theta=0$ is given in Table 2. Table 2 indicates that the convergence of the values in the interior points is very rapid and that the convergence of $\sigma_{\theta \theta}$ and $\sigma_{z z}$ at $z=a$ is however slightly slow. For the magnesium crystal and the isotropic material, the distributions of $\sigma_{r r}, \sigma_{\theta \theta}, \sigma_{z z}$ and $\sigma_{z r}$ in the planes of $\theta=0$ and $\theta=\pi$ are shown in Figs.2, 3, 6 and 7 . 
Table 1 Values of elastic constants $c_{i j}$

(in units of 10cira).

\begin{tabular}{llllll}
\hline \multicolumn{1}{c}{ Material } & $c_{44}$ & $c_{11}$ & $c_{33}$ & $c_{12}$ & $c_{13}$ \\
\hline Magnesium crystal & 1.64 & 5.97 & 6.17 & 2.62 & 2.17 \\
Cadmium crystal & 1.56 & 11.0 & 4.69 & 4.04 & 3.83 \\
Isotropy $(v=0.25)$ & 1.0 & 3.0 & 3.0 & 1.0 & 1.0 \\
\hline
\end{tabular}

Table 2 Check for convergence of $\sigma_{r r}, \sigma_{\theta \theta}$ and $\sigma_{z z}$ at $\theta=0$. (Magnesium, $h / a=1.0, d / a=0.3, \omega=0.3 \pi$ ).

\begin{tabular}{|c|c|c|c|c|c|c|c|c|}
\hline \multicolumn{3}{|c|}{ Number of terms } & \multicolumn{2}{|c|}{$-\sigma_{r r} / q$} & \multicolumn{2}{|c|}{$-\sigma_{\theta \theta} / q$} & \multicolumn{2}{|c|}{$-\sigma_{z z} / q$} \\
\hline$m$ & $s$ & $n$ & $\begin{array}{l}r=0.4 a \\
z=0.8 a\end{array}$ & $\begin{array}{l}r=a \\
z=0.8 a\end{array}$ & $\begin{array}{l}r=0.2 a \\
z=0.8 a\end{array}$ & $\begin{array}{l}r=0.2 a \\
z=a\end{array}$ & $\begin{array}{l}r=0.2 a \\
z=0.8 a\end{array}$ & $\begin{array}{l}r=0.2 a \\
z=a\end{array}$ \\
\hline 20 & 20 & 20 & 0.0673 & 0.0001 & 0.0180 & 0.7084 & 0.5368 & 0.9074 \\
\hline 30 & 30 & 30 & 0.0673 & 0.0002 & 0.0180 & 0.7257 & 0.5368 & 1.0421 \\
\hline 40 & 38 & 38 & 0.0673 & -0.0005 & 0.0180 & 0.7219 & 0.5368 & 1.0036 \\
\hline 50 & 50 & 50 & 0.0673 & 0.0001 & 0.0180 & 0.7247 & 0.5368 & 1.0374 \\
\hline
\end{tabular}

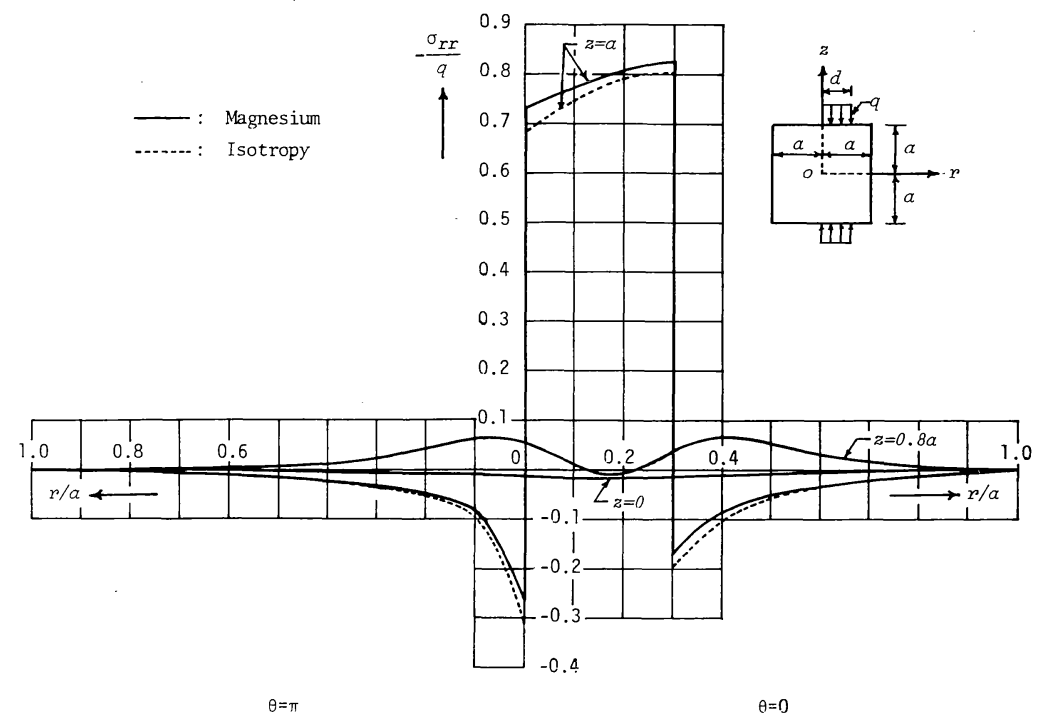

Fig.2 Distribution of $\sigma_{r r}$ in planes of $\theta=0$ and $\theta=\pi$. (Magnesium and Isotropy, $h / a=1.0, d / a=0.3, \omega=$ $0.3 \pi)$.

Fig.2 shows that the decay of $\sigma_{r r}$ along the $r$ direction is rapid and that the value at the end face $(z=a)$ becomes discontinuous at the boundary $(r=$ 0 and $r=0.3 a$ ) of the sectorial load. The value at the end face was calculated by the method proposed by Saito ${ }^{9}$. Furthermore, the values in the points away from the end face are small due to the small loading area. Fig.3 shows that the value of $\sigma_{\theta \theta}$ at $z=a$ becomes discontinuous at $r=0$ and $r=$ $0.3 a$ like $\sigma_{r r}$ and that the values in the points away from $z=a$ are small. Figs.4 and $\mathbf{5}$ show the distributions of $\sigma_{r r}$ at $r=0.4 a$ and $z=0.8 a$ and $\sigma_{\theta \theta}$ at $r=0.2 a$ and $z=0.8 a$ along the $\theta$-direction for the magnesium crystal. It is shown that the decay of $\sigma_{r r}$ and $\sigma_{\theta \theta}$ along that direction is rapid. Fig. 6 shows that the value of $\sigma_{z z}$ at $z=0.8 a$ becomes small positive one at the side surface $(r=a)$ and that the values in the points away from $z=a$ are comparatively large. Fig. 7 shows that the values of $\sigma_{z r}$ are less than the normal stresses and that the values in the points near $z=a$ become larger at $r=0.3 a$. In Fig. 7 , the values in the plane of $\theta=\pi$ are drawn in the inverse sign. Tables 3 and 4 indicate comparisons of the displacement and stress values among the magnesium crystal, the cadmium crystal and the isotropic material, respectively. Table 3 shows 


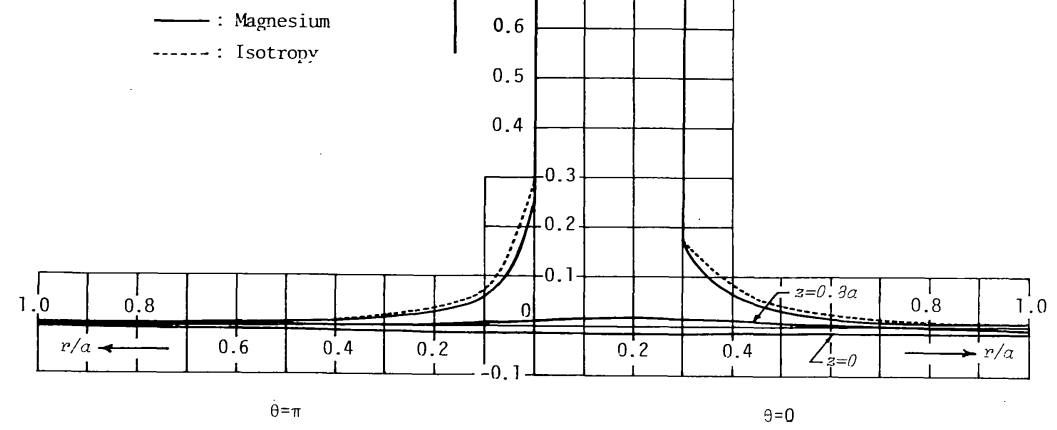

Fig.3 Distribution of $\sigma_{\theta \theta}$ in planes of $\theta=0$ and $\theta=\pi$. (Magnesium and Isotropy, $h / a=1.0, d / a=0.3, \omega=$ $0.3 \pi)$.

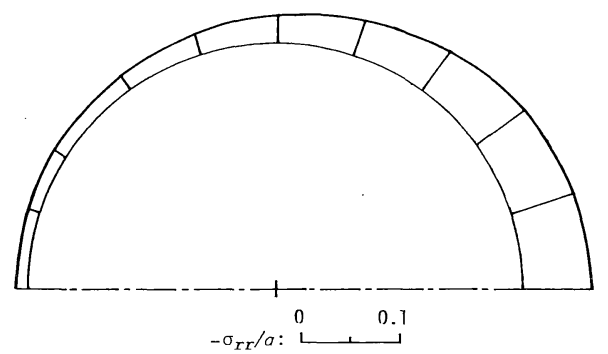

Fig.4 Distribution of $\sigma_{r r}$ along $\theta$-direction. (Magnesium, $r=0.4 a, z=0.8 a$ ).

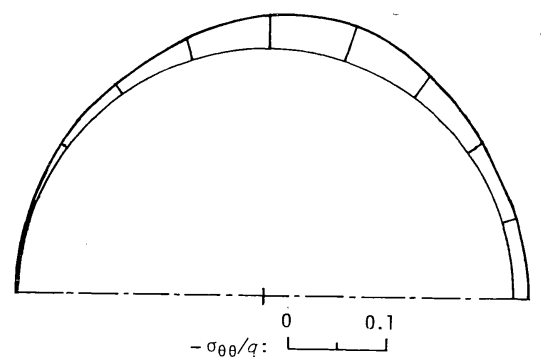

Fig.5 Distribution of $\sigma_{\theta \theta}$ along $\theta$-direction. (Magnesium, $r=0.2 a, z=0.8 a$ ).

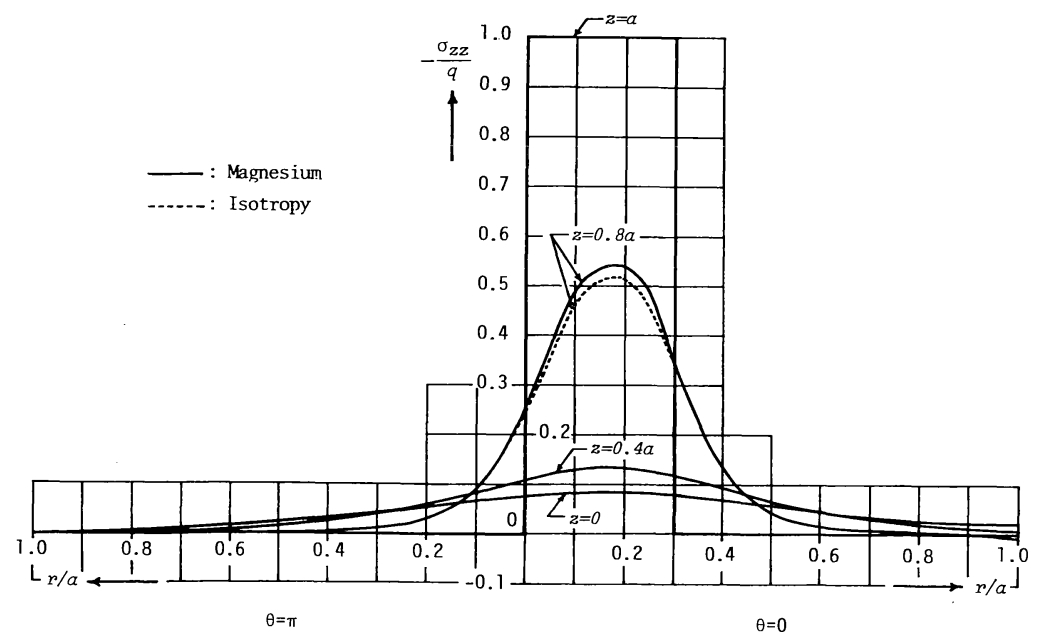

Fig.6 Distribution of $\sigma_{z z}$ in planes of $\theta=0$ and $\theta=\pi$. (Magnesium and Isotropy, $h / a=1.0, d / a=0.3, \omega=$ $0.3 \pi)$. 


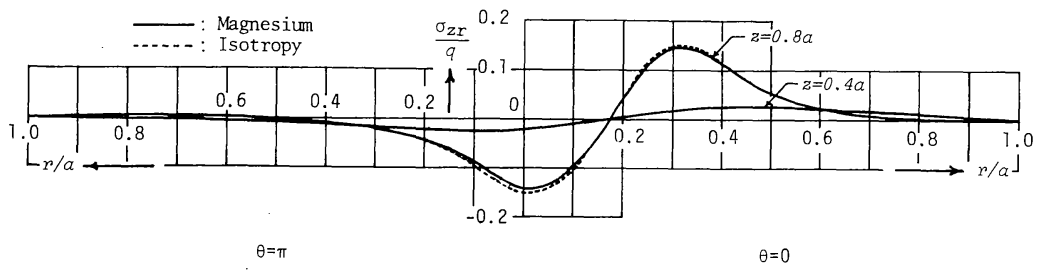

Fig.7 Distribution of $\sigma_{z r}$ in planes of $\theta=0$ and $\theta=\pi$. (Magnesium and Isotropy, $h / a=1.0, d / a=0.3, \omega=$ $0.3 \pi)$.

Table 3 Comparisons of displacement values $(r=0.2 a, \theta=0.2 \pi, z=0.8 a)$.

\begin{tabular}{lccc}
\hline Material & $u_{r} / \frac{q a}{c_{11}}$ & $u_{\theta} / \frac{q a}{c_{11}}$ & $u_{z} / \frac{q a}{c_{11}}$ \\
\hline Magnesium crystal & 0.00657 & 0.0125 & -0.157 \\
Cadmium crysta1 & 0.0191 & 0.0259 & -0.358 \\
Isotropy & 0.00634 & 0.0115 & -0.146
\end{tabular}

that the values of $u_{r}, u_{\theta}$ and $u_{z}$ in the cadmium crystal are more than those in the isotropic material by $201.3 \%, 125.2 \%$ and $145.2 \%$, respectively, but the values in the magnesium crystal differ slightly from those in the isotropic material. Table 4 shows that the values of $\sigma_{\theta \theta}$ in the magnesium and cadmium crystals are less and more than that in the isotropic material by $7.4 \%$ and $85.8 \%$, respectively.

\section{CONCLUSIONS}

Axially asymmetric stresses in a transversely isotropic, short cylinder subjected to sectorial pressures on the end faces were analyzed by the generalized Elliott solution. The method of analysis for the three-dimensional, asymmetric stress problem of the short cylinder was stated briefly. From the results of the numerical calculations for the short cylinder, the following conclusions may be drawn :

(1) The values of $\sigma_{r r}$ and $\sigma_{\theta \theta}$ at the end face are discontinuous at the boundary of the sectorial load.

(2) The decay of $\sigma_{r r}$ and $\sigma_{\theta \theta}$ along the radial and circumferential directions is rapid.

(3) The values of $\sigma_{r r}$ and $\sigma_{\theta \theta}$ in the points away from the end face are small due to the small loading area.

(4) The values of $\sigma_{z z}$ in the points near the end face are positively small at the side surface.

(5) The value of $u_{r}$ at $r=0.2 a, \theta=0.2 \pi$ and $z$ $=0.8 a$ in the cadmium crystal is $201.3 \%$ more than that in the isotropic material with Poisson's ratio 0.25 .

(6) The value of $\sigma_{\theta \theta}$ at $r=0.2 a, \theta=0$ and $z=$
Table 4 Comparisons of stress values $(r=0.2 a, \theta=0, z=0.9 a)$.

\begin{tabular}{lcccc}
\hline Material & $\sigma_{x r / q}$ & $\sigma_{\theta \theta / q}$ & $\sigma_{z z / q}$ & $\sigma_{z r / q}$ \\
\hline Magnesium crysta1 & -0.114 & -0.163 & -0.833 & 0.0628 \\
Cadmium crysta1 & -0.203 & -0.327 & -0.749 & 0.0816 \\
Isotropy & -0.121 & -0.176 & -0.826 & 0.0674 \\
\hline
\end{tabular}

$0.9 a$ in the cadmium crystal is $85.8 \%$ more than that in the isotropic material.

(7) The differences in the displacement and stress values between the magnesium crystal and the isotropic material are narrow.

(8) The differences in the displacement and stress values between the transversely isotropic and isotropic materials grow wider as those of the values of the elastic constants become wider.

\section{REFERENCES}

1) Levine, H.S. and Klosner, J.M. : Transversally isotropic cylinders under band loads, Proc. ASCE, J. Eng. Mech. Div., Vol.93, No.EM3, pp.157 174, 1967.

2) Atsumi, A. and Itou, S. : Stresses in a transversely isotropic circular cylinder having a spherical cavity, Trans. ASME, J. Appl. Mech., Vol.41, No.2, pp.507 511, 1974.

3) Mirsky, I. : Wave propagation in trasversely isotropic circular cylinders, J. Acoust, Soc. Am., Vol.37, No.6, pp.1016 1021, 1965.

4) Chen, W.T. : On some problems in transversely isotropic elastic materials, Trans. ASME, J. Appl. Mech., Vol.33, No.2, pp.347 355, 1966.

5) Zureick, A.H. : Spheroidal cavity with prescribed asymmetric displacements in three-dimensional transverse isotropy, Proc. ASCE, J. Eng. Mech., Vol.115, No.11, pp.2427 2439, 1989.

6) Okumura, I.A. : Generalization of Elliott's solution to transversely isotropic solids and its application, Proc. JSCE, Struct. Eng./Earthquake Eng., Vol.4, No.2, pp.401s 411s, 1987.

7) Okumura, I.A. : Stresses in a transversely isotropic, short hollow cylinder subjected to an outer band load, Ing.Arch., Vol.59, No.4, pp.310 324, 1989.

8) Huntington, H.B. : The elastic constants of crystals, In : 
Seitz, F. and Turnbull, D. (eds.) Solid State Physics, Vol.7, p.213, Academic Press, 1957.

9) Saito, H. : The axially symmetrical deformation of a short circular cylinder and a circular disk, Trans. Jpn. Soc.
Mech. Eng., Vol.18, No.68, pp.21 28, 1952 (in Japanese).

(Received September 8, 1993)

\section{端面に扇形分布圧力を受ける横等方性短円柱の非軸対称応力 \\ 奥村 勇}

両端面に扇形分布の一様荷重を受ける横等方性短円柱の非軸対称，3 次元応力が解析 されている. 解析には，一般化された Elliott の解が用いられている．この解は，短円 柱の両端面および側面の境界条件を満足できる二種類の異なった弾性解をもたらす．数 值計算においては，横等方性材料の一例として，マグネシウムおよびカドミウムの単結 晶が取り扱われ，更に，ポアソン比が 0.25 の等方性材料も取り扱われている.これら の材料に関する応力および変位の值が，図および表に示されている．等方性材料との比 較により，異方性の効果が調べられている。 